gehen. Dabei zeigte sich, daß die Umsetzung der hier skizzierten Postulate sowohl an Auftragnehmer als auch Auftraggeber einer solchen institutionenbezogenen Politikberatung hohe Anforderungen stellt. Eine transdisziplinäre Institutionenperspektive erwies sich auch in diesem Projekt als ein Forschungs- und Beratungsfeld von hoher praktischer und theoretischer Relevanz, dem es sich auch in Zukunft zu stellen lohnt.

\section{Anmerkungen}

(1) Ein Beispiel bietet die von den Autoren verfaßte Studie "Institutionelle Reformen für eine Politik der Nachhaltigkeit“ für die Enquete-Kommission „Schutz des Menschen und der Umwelt". Die Studie erscheint als: Arbeitsgemeinschaft IWÖ/IFOK: Institutionelle Reformen für eine Politik der Nachhaltigkeit im Frühjahr 1998 im Springer Verlag (Berlin, New York). Eine Zusammenfassung der Studie findet sich in GAIA 6/3 (1997), S.182-197.

(2) Vgl. zur Neuen Institutionenökonomik z.B. R. Richter, E. Furubotn: Neue Institutionenökonomik. Tübingen 1996.

(3) H. Diett: Institutionen und Zeit. Tübingen 1983, S. 71 ff.

(4) D. Kiwit, S. Voigt: Überlegungen zum institutionellen Wandel unter der besonderen Berücksichtigung des Verhältnisses interner und externer Institutionen, in: Ordo, Jg. 46 (1995),

S. 117-147.

(5) Zu einer solchen spielbezogenen Interpretation gesellschaftlicher Zusammenhünge vgl. insbesondere die Analyse von A. Burkard: Markt als Gesellschaftsspiel. Bern 1997 (siehe auch Rezension in diesem Heff).

(6) A. Giddens: Die Konstitution der Gesellschaft. Frankfurt und New York 1988.

(7) Vgl. z.B. D. Messner: Die Netzwerkgesellschaft. Köln 1995.

(8) Vgl. zu diesen Vorschlägen die Enquete-Studie „Institutionelle Reformen" a.a.0.

\section{Die Autoren}

Dr. Hans Peter Meister, Dipl. Pol. Peter Henning Feindi, Jochen Tscheulin, MA, Jürgen Wüst, MA, alle Institut für Organisationskommunikation (IFOK), Bensheim.

Dr. Jürg Minsch, Lic. oec. Tobias Schulz, beide Institut für Wirtschaft und Ökologie an der Universitüt St. Gallen (IW0̈-HSG).

Dr. Uwe Schneidewind, Lehrstuhl für Produktion und Umwelt, Universität Oldenburg.

Kontakt: Uwe Schneidewind, Universitüt Oldenburg, FB 4, Birkenweg 5, D-26111 Oldenburg, Tel: 0441/798-8255, -8300 ,

e-mail: schwind@hrzl.uni-oldenburg.de.

Herausforderungen für eine transdisziplinäre ökologische Wirtschaftslehre

\title{
Zwischen Arbeitsmarkt und wissenschaftlichen Idealen
}

\author{
In den letzten Jahren sind einige ökologische Studienangebote mit inter- \\ oder transdisziplinärem Anspruch in den Wirtschaftswissenschaften \\ entstanden. Die Autoren beleuchten insbesondere vor ihren in St. Gallen und \\ Oldenburg gemachten Erfahrungen, welche Herausforderungen sich solchen \\ Studiengängen stellen und wie diesen zu begegnen ist.
}

$\mathrm{N}$ Von Christoph Schwarzer und Uwe Schneidewind schaften (1) ziehen seit einigen Jahren auch viele wirtschaftswissenschaftliche Fakultäten mit der Einrichtung interdisziplinärer ökologischer Studienangebote nach (2). In einigen Fällen beschränkt sich dies auf einen im wesentlichen betriebs- und volkswirtschaftlichen Kern, in anderen Fällen erstreckt sich das Studienangebot gleichberechtigt auf natur-, wirtschafts- und geisteswissenschaftliche Bezüge wie z.B. beim Studiengang Umweltwissenschaften an der Universität Lüneburg. Von Wissenschaftlern und Praktikern wird zunehmend der Vorwurf laut (3), hier würden Absolventen zur Arbeitslosigkeit ausgebildet, da diese Studiengänge von ,allem ein bißchen, aber nichts richtig" vermittelten. Dies sei gerade angesichts der wieder geringeren Aktualität des Themas "Ökologie“" für die Arbeitsmarktchancen von Absolventen besonders bedenklich.

Dieser Gefahr ist nur dadurch zu begegnen, daß die spezifischen Herausforderungen, vor denen solche interdisziplinären Studiengänge stehen, genau analysiert und die Studienangebote entsprechend darauf ausgerichtet werden.

\section{- Externe und interne Herausforderungen}

Die angespannte Arbeitsmarktlage führt dazu, daß sich in den letzten Jahren die Arbeitsmarktchancen für Volks- und Betriebswirte erheblich verschlechtert haben. Die Studierenden reagieren darauf, indem sie sich in ihren Studienvertiefungen auf klassische (betriebswirtschaftliche) Kernfächer wie z.B. Rechnungswesen/ Controlling, Marketing, Wirtschaftsinformatik konzentrieren, bei denen die Arbeitsmarktchancen noch relativ gut sind. Ein
Studienschwerpunkt "Ökologie" hat deswegen mit der Gefahr zu kämpfen, daß er als karrierehinderlich und arbeitsplatzabträglich angesehen wird.

Viele der heutigen Stellen und Aufgaben im Bereich des Umweltmanagements erscheinen den Studierenden als wenig attraktiv, da sie in vielen Unternehmen immer noch eine Karrieresackgasse bedeuten, d.h. wenige Weiterentwicklungsmöglichkeiten öffnen. Zudem ist die Konkurrenz von Bewerbern aus Nachbardisziplinen (insbesondere aus den Ingenieur- und Naturwissenschaften) sehr hoch.

Aufgrund der Tatsache, daß neben einem ökologischen Schwerpunkt, der einen Großteil des frei wählbaren Hauptstudiumspensums umfaßt (in Oldenburg oder St. Gallen z.B. 24 Semesterwochenstunden), für die Studierenden auch die Möglichkeit besteht, Ökologie lediglich durch kleinere Wahlblöcke oder Einzelveranstaltungen abzudecken, entsteht an den Universitäten eine interne Wettbewerbssituation in der ökologischen Ausbildung: Studierende mit ökologischem Interesse sind nicht darauf angewiesen, den ökologischen Schwerpunkt zu wählen, sie können dieses Interesse auch durch die Belegung anderer - weniger aufwendiger - Veranstaltungen befriedigen. Angesichts der oben beschriebenen externen Herausforderungen besteht die Gefahr, daß sich viele Studierende für eine solche Variante entscheiden.

Das Studienangebot im ökologischen Schwerpunkt ist - wie in den meisten heute angebotenen ökologischen Vertiefungen - in erster Linie durch die Angebote der daran interessierten Dozenten geprägt. Es ist deswegen von der Konstruktion angebots-und nicht nachfrageorientiert. Es besteht die Gefahr, daß eine Summe von interessanten Einzelaspekten im ökologischen Schwerpunkt vermittelt wird, eine inhaltliche 
und auf die späteren Arbeitsmarktchancen der Absolventen ausgerichtete- Integration und Fokussierung der Aspekte aber nicht erfolgt. Aus den skizzierten Herausforderungen ergeben sich insbesondere drei Konsequenzen:

1. Ein ökologischer Studienschwerpunkt benötigt ein eigenständiges Profil. Den Studierenden muß klar sein, was genau der Mehrwert dieses Studienschwerpunktes im Vergleich zur Belegung von ökologischen Einzelveranstaltungen oder Wahlblöcken darstellt. Dieses Profil muß so angelegt sein, daß es neben einer fundierten ökologischen Ausrichtung die Vielschichtigkeit der ökologischen Problematik nutzt, um Generalisten zu fördern, die sich schnell in neue, komplexe Zusammenhänge einfinden können.

2. Es muß Klarheit über die späteren beruflichen Einsatzfelder der Absolventen bestehen. Der Studienschwerpunkt muß so konzipiert sein, daß er auf diese Einsatzfelder vorbereitet. 3. Die Nachfrageorientierung muß sich auch auf studentischer Seite wiederfinden, d.h. der Studiengang muß genügend Freiheiten und Unterstïtzung für die Kultivierung und Erprobung eigener Interessen und Projekte bieten. Nur aus diesen Erfahrungen speist sich Selbstvertrauen und Motivation, die auch mit einem etwas ungewöhnlichen Zugang Erfolg verspricht.

\section{Ein angemessenes Leitbild}

Ein mögliches Leitbild für einen transdisziplinären ökologischen Schwerpunkt könnte vor dem Hintergrund der dargestellten Herausforderungen wie folgt aussehen:

Das Studium des ökologischen Studienschwerpunktes soll Akteure des ökologischen Strukturwandels ausbilden. Es wendet sich an Studierende, die später in Unternehmen, in Verbänden, in der Politik, in Umweltschutzorganisationen und anderen NGO`s Beiträge zur ökologischen Umgestaltung von Wirtschaft und Gesellschaft leisten. Die Studierenden sollen mit dem Studium die Grundlage dafür erhalten, ökologische Herausforderungen in Wirtschaft und Gesellschaft zu erkennen, $\mathrm{zu}$ analysieren und in konkretes Handeln umzusetzen.

Ein solches Leitbild geht über Berufsfelder wie dasjenige eines Umweltbeauftragten hinaus. Es erkennt, daß der ökologische Wandel mit zahlreichen gesellschaftlichen und wirtschaftlichen Umbrüchen einhergeht, die konkrete wirtschaftliche Chancen eröffnen. Absolventen eines entsprechenden Studienschwerpunktes müssen in besonderer Weise befähigt sein, solche Herausforderungen in bestehenden Organisationen, durchaus aber auch durch die Gründung eigener Unternehmen wahrzunehmen.

Konkrete Tätigkeitsfelder ergeben sich für die Absolventen demnach u.a. in

- Unternehmen ökologisch betroffener Branchen (z.B. Automobil, Chemie, Energie, Mineralöl, Elektronik, Umwelttechnik), dort insbesondere in den Bereichen der strategischen Planung, des Marketings, des Produktions- und Logistikmanagements,

- Unternehmen im Banken- und Versicherungssektor (insbesondere Rückversicherung),

- Unternehmensverbänden und

- Gewerkschaften, vor allem in ökologisch betroffenen Branchen,

- (strategischen) Unternehmensberatungen,

- Umweltministerien und Umweltbehörden,

- Hochschulen und Forschungseinrichtungen,

- Umweltschutzorganisationen,

- Entwicklungshilfeinstitutionen und -organisationen,

- internationalen Gremien (EU, Weltbank etc.),

- als Existenzgrïnder in neuen, durch den ökologischen Strukturwandel aufbrechenden Marktnischen und in

- alten und neuen Medien.

\section{Qualifikationen erforderlich}

Die Einlösung der dargestellten Ausbildungsziele bedingt ein breites Spektrum an Qualifikationen, das sowohl der Studiengang als auch die den Studiengang begleitende Aktivitäten den Studierenden vermitteln müssen. Dabei ist bedeutsam, daß der ökologische Studienschwerpunkt diese Qualifikationen im Vergleich $\mathrm{zu}$ anderen Studiengängen (bei denen z.B. lediglich ein ökologisches Wahlpflichtfach belegt wird) in besonders geeigneter Weise vermittelt. Insbesondere stellt der Zusammenhalt zwischen den Studierenden des Studienganges, der durch besondere Projektveranstaltungen, durch Exkursionen und gemeinsames Arbeiten an Studienarbeiten gefördert wird, einen Bonus dar.

Inbaltlich muß ein entsprechender Studiengang- bzw. -schwerpunkt folgende Qualifikationen unbedingt abdecken:

- Umfassendes gesellschaftstheoretisches Orientierungswissen, das es den Studierenden erlaubt, die mit der ökologischen Frage verbun- denen gesellschaftlichen, politischen und wirtschaftlichen Umbrüche einzuordnen und zu verstehen (Umweltrecht, Umweltpolitik/Sozialwissenschaften, Volkswirtschaftslehre).

- Betriebswirtschaftliches Grundlagenwissen, das die Studierenden befähigt, die strategischen und konkreten Erfolgsauswirkungen von ökologischen Herausforderungen für Unternehmen (aber auch andere gesellschaftliche Akteure) zu ermitteln und geeignete Gestaltungskonzepte als Antwort zu entwickeln (u.a. unter Rückgriff auf die Erkenntnisse der strategischen Managementlehre und des Marketings).

- Naturwissenschaftliches Grundlagenwissen, das zumindestens exemplarisch auch in Laborversuchen und Diskussionen mit Naturwissenschaftlern erprobt ist und die Studierenden in die Lage versetzt, sich naturwissenschaftlichen Fragestellungen adäquat zu nähern und fundiert mit Naturwissenschaftlern und Ingenieuren zu kommunizieren.

Neben den inhaltlichen Qualifikationen, die die Studierenden in die Lage versetzen sollen, komplexe gesellschaftliche und ökonomische Problemlagen adäquat $\mathrm{zu}$ analysieren, sollte das Studium methodische Qualifikationen vermitteln, die es den Absolventen später erleichtern, die Ergebnisse ihrer Analysen in konkretes Handeln umzusetzen. Hierzu gehören u.a. Kompetenzen in den Bereichen Kommunikation, Präsentation, Moderation, Teamarbeit, Projektplanung und Existenzgründung.

Darüber hinaus sollte das Studium den Erwerb von Zusatzqualifikationen unterstïtzen, die sowohl die Arbeitsmarktchancen als auch die persönlichen Qualifikationen der Studierenden erhöhen. Hierzu gehören

- eigenorganisiertes und -verantwortetes Tätigwerden in studentischen Initiativen ,

- gezielte Studien-Auslandsaustausche und Auslandspraktika (mit Ökologiebezug) und

- die aktive Nutzung neuer Medien im Rahmen des Studiums.

\section{Projektstudium als ein Weg}

Für die Vermittlung dieses breiten Fähigkeitsspektrums bietet insbesondere ein gut betreutes Projektstudium eine hervorragende Grundlage. An der Universität Oldenburg wird dies z.B. dadurch umgesetzt, daß zwei wissenschaftliche Mitarbeiter sich insbesondere der Koordination der im Studienschwerpunkt erbrachten Projekte widmen. Neben der Qualifikationsvermittlung im eigentlichen Studiengang ist es wichig, daß die am Studi- 
enangebot beteiligten Lehrstiihle die Berufsqualifikation der Absolventen durch weitere Maßnahmen unterstïtzen. Hierzu gehören

- innovative Lehrstuhlumfelder, in denen die Unterstiitzung bei studentischer Existenzgrïndung, die Nutzung von neuen Medien, die Arbeit mit modernen Didaktik- und Moderationskonzepten zur Selbstverständlichkeit gehört und die den Studierenden so immer wieder Anregungen zum Nachahmen und zur Weiterentwicklung geben,

- aktive Kontaktaufnahme der Lehrstïhle mit möglichen Arbeitgebern und Arbeitsfeldern der späteren Absolventen, um einerseits für die spezifischen durch den Studiengang geworbenen Qua* lifikationen zu werben und andererseits das Ausbildungsprofil vor dem Hintergrund aktueller Ausbildungserfordernisse weiterzuentwickeln.

- gezielte Werbung und Auswahl von Studierenden für den Studiengang, um einen möglichst hohen Anteil an qualifizierten und motivierten Studierenden für den Studienschwerpunkt zu gewinnen.

- Kooperationen mit anderen Fachbereichen und Initiativen auf Universitätsebene, um das breite und qualifizierte Potential, das eine Universität im ökologischen Bereich hat, den Studierenden leichter zugänglich zu machen.

Eine transdisziplinäre ökologische Wirtschaftslehre ist keine Karrieresackgasse, wenn sie strategisch geeignet umgesetzt wird.

\section{Anmerkungen}

(1) Als prominentestes Beispiel kann hier der Studiengang Umweltnaturwissenschaften der ETH Zürich genannt werden. (2) Vgl. hierzu auch die Vorstellung des Diplomstudienganges Ökonomie mit ökologischen Schwerpunkt an der Universität Oldenburg in der Ausgabe Nr. 5/1997 von Ökologisches Wirtschaften sowie den Beitrag von Wenke in diesem Heft.

(3) So erst kürzlich wieder in einem Beitrag auf der Hochschulseite der Frankfurter Allgemeinen Zeitung vom 2.1.1998.

\section{Die Autoren}

Dipl. - Oec. Chrisioph Schwarzer ist einer der beiden Koordinaloren für den Diplomstudiengang Ökonomie mit ökologischen Schwerpunkt an der Carl-vonOssiełzky-Universität Oldenburg

Dr. Uwe Schneidewind vertritt die Professur für „Produktion und Umwelt" an der Universitüt Oldenburg und war zuvor an der Universität St. Gallen tätig. Kontakt: Christoph Schwarzer, Universität Oldenburg, Fachbereich 4, Birkenweg 5, D-26111 Oldenburg, Tel::0441/798-8265, e-mail: schwarz@unioldenburg.de. Dort ist auch eine Broschüre zum Diplomstudiengang „Ökonomie mit ökologischen Schwerpunkt" erhällich.

Transdisziplinarität in der Unternehmenspraxis

\section{Immer bedeutenderer Schlüsselfaktor}

\section{Transdisziplinäre Wirtschaftsforschung ist nicht nur eine wissenschaftliche Trockenübung. Sie gewinnt zunehmend auch in der Unternehmensentwicklungs- und -berafungspraxis an Bedeutung. Der vorliegende Beitrag illustriert dies an zwei Beispielen aus der Marktentwicklung im Bereich Öko-Textilien sowie der Einführung von Integrierten Managementsystemen bei der Hoechst AG.}

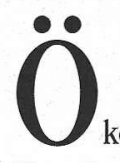
Von Arnt Meyer und
Frank Riemenschneider kologische Produkte (1) sind derzeit überwiegend in Nischenmärkten zu finden, die Durchdringung von „Massenmärkten“ gelingt nur in Ausnahmefällen. Diese Aussage ist zunächst banal, spiegelt aber die Entfernung der Wirtschaft von ökologischen Strukturen realistisch wider. Für die Initiierung ökologischer Wandlungsprozesse auf Märkten sind viele Faktoren relevant. Ein wesentliches Kriterium scheint der Versuch einer Beeinflussung von Konsumenten zu sein, der in der Regel vom unternehmerischen (Öko-) Marketing vorgenommen wird. Ökologisches Marketing, vor allem die Kommunikation mit den Konsumenten, wird häufig mit informations- ökonomischen Erkenntnissen begründet (2). Demnach liegen die Gründe für eine geringe Marktdurchdringung mit ökologischen Produkten u.a. in Informationsdefiziten. Es wird angenommen, Konsumenten seien ungenügend über die ökologischen Eigenschaften von Produkten informiert und gelangten aus diesem Grund nicht zu ökologisch sinnvollen Entscheidungen. Anbieter ökologischer Erzeugnisse sind da- durch gezwungen, ihre Produktmerkmale verstärkt $z u$ kommunizieren (Signalling). Daß diese Erkenntnisse die Kommunikationspraxis ökologisch bewußter Unternehmen prägen, zeigt die zunehmend zu beobachtende Volldeklaration ökologischer Erzeugnisse. Auch hinsichtlich der Werbe-Darstellung werden ökologisch glaubwürdige Formen (Verzicht auf Hochglanzpapier, keine schrillen Werbeclips, etc.) sowie sachliche und informative Werbebotschaften empfohlen (3). Eine nähere Betrachtung zeigt jedoch, daß diese enge ökonomische Sichtweise für die Initiierung ökologischer Wandlungsprozesse ungeeignet ist.
Zum einen liegt einer solchen Handlungsempfehlung ein verkürztes, mechanistisches Kommunikationsverständnis zu Grunde. Kommunikative Prozesse werden auf ein Sender-Empfänger-Modell verkürzt, bei dem der Empfänger von Informationen (hier der Konsument) die ausgesendeten Signale einszu-eins übernimmt und folglich im Sinne des Senders handelt (4). Zudem werden Märkte zu einseitig betrachtet, da davon ausgegangen wird, daß die Konsumenten in mehr oder weniger gleichem Umfang für ökologische Belange sensibilisierbar sind. Am Institut für Wirtschaft und Ökologie der Universität St. Gallen (IWÖHSG) wird deshalb in Kooperation mit Unternehmen der deutschen und schweizerischen Bekleidungsbranche ein anderer, den interdisziplinären Notwendigkeiten einer ökologischen Marktentwicklung angemessener Weg verfolgt.

\section{Erweitertes \\ Kommunikationsverständnis}

Den Ausgangspunkt dieser Herangehensweise bildet eine soziologische Betrachtung der Rahmenbedingungen von Märkten. Die (industrialisierten) Gesellschaften tendieren - vereinfacht gesagt - aufgrund von Aspekten wie Enttraditionalisierung (5), Multioptionalisierung (6) oder Erlebnisorientierung (7) in Richtung einer starken gruppen- und individualspezifischen Fragmentierung. Daraus folgt letztlich, daß es nicht den Massenmarkt für Produkte gibt, sondern nur stark fragmentierte Teilmärkte.

Auch kommunikationstheoretisch wird mit einem anderen Ansatz gearbeitet. Kommunikation ist ein sozialer Prozeß, in dem die beteiligten Akteure mittels interpretierbarer Worte und Taten Informationen mit einer bestimmten Intention zu transportieren versuchen. Dieser 
(c) 20I0 Authors; licensee IÖW and oekom verlag. This is an article distributed under the terms of the Creative Commons Attribution Non-Commercial No Derivates License (http://creativecommons.org/licenses/by-nc-nd/3.o/), which permits unrestricted use, distribution, and reproduction in any medium, provided the original work is properly cited. 